\section{Dilemas éticos acerca de la revelación de errores médicos a los pacientes}

\author{
SEBASTIÁN LAVANDEROS ${ }^{1,2}$, JUAN PEDRAZA ${ }^{1,3}$, \\ MOISÉS RUSSO N. ${ }^{1}$, SOFÍA P. SALAS ${ }^{1}$

\section{Ethical dilemmas about disclosure of errors in medicine}

Since the publication of the Institute of Medicine's report "To Err is Human: Building a Safer Health System" awareness of the importance of medical errors has increased. These are a major cause of morbidity and mortality and recent studies suggest that they can be the third leading cause of death in the United States. Difficulties have been identified by health personnel to prevent, detect and disclose to patients the occurrence of a medical error, an also to report them to the appropriate authorities. Although human error cannot be eliminated, it is possible to design safety systems to mitigate their frequency and consequences. Our goal is to provide an update on the major concepts related to medical errors, a review of Chilean legislation on the subject, and a bioethical analysis on the principles involved, along with a management proposal. We propose mandatory reporting of errors to the health institution where they occur, to serve as a measure of personal and team learning, and to disclose the error to patients, depending on their own preferences.

(Rev Med Chile 2016; 144: 1191-1198)

Key words: Ethics; Disclosure; Medical Errors; Patient Safety; Risk Management.
${ }^{1}$ Escuela de Medicina, Universidad Diego Portales. Santiago, Chile.

${ }^{2}$ Médico EDF, Hospital de Loncoche, Loncoche, Chile. ${ }^{3}$ Médico EDF, CESFAM Bernardo Leighton, Puente Alto, Santiago, Chile.

Conflictos de interés: Ninguno que declarar. Este trabajo es parte de un ensayo realizado en la asignatura de Bioética Clínica II, Escuela de Medicina UDP.

Recibido el 25 de agosto de 2015, aceptado el 26 de julio de 2016

Correspondencia a: Dra. Sofía P. Salas Ejército 260; Santiago, Chile. sofia.salas@udp.cl

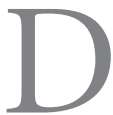
esde la publicación del reporte del Instituto de Medicina (IOM) de los Estados Unidos de Norteamérica (EE. UU.) "To Err is Human: Building a Safer Health System”, en el año 1999, se ha observado mayor conciencia respecto de los errores en la atención sanitaria y su impacto en la salud de las personas ${ }^{1}$. A modo de ejemplo, una búsqueda simple en la base de datos PubMed de artículos con las palabras "medical error" en su título, resumen, términos MESH o palabras claves nos muestran 4 artículos publicados el año 1998, 35 artículos en el año 2000 y 123 artículos el año 2015. Dicho informe reconoce que los seres humanos, independiente del tipo de trabajo, cometen errores, pero que estos pueden ser prevenidos si se diseñan sistemas que dificulten a las personas realizar una acción de manera incorrecta y que promuevan hacerla de manera adecuada. Un trabajo publicado recientemente señala que los errores médicos serían la tercera causa de muerte en los EE. UU. ${ }^{2}$, sugiriendo que los datos del IOM han subestimado la importancia del error como causa de muerte.

Dada la importancia del error en la atención de salud como causa de morbimortalidad y la dificultad que tenemos para prevenirlos, detectarlos y revelarlos al paciente y reportarlos a las autoridades correspondientes, en el presente artículo realizaremos un análisis de los principios bioéticos involucrados, junto a una propuesta de manejo. Usaremos de forma genérica el concepto de "error médico" para referirnos no sólo a los errores cuya responsabilidad es de los médicos, sino que aquellos ocasionados por cualquier integrante del equipo de salud. 


\section{Concepto de error en medicina}

El informe del IOM define al error médico como la "la falla de una acción planeada en completarse como se pretendía, o la aplicación de un plan equivocado para alcanzar un objetivo"'. En el año 2009, la Organización Mundial de la Salud publicó un marco conceptual de la clasificación internacional para la seguridad del paciente, que define un concepto de error similar al del informe del $\mathrm{IOM}^{3}$, y que permite comparar datos sobre seguridad del paciente en distintos ámbitos, países y regiones y también analizar los papeles que desempeñan tanto los factores humanos como de los sistemas en la seguridad del paciente, utilizando una nomenclatura común. Este documento define a incidente relacionado con la seguridad del paciente como "un evento o circunstancia que podría haber ocasionado u ocasionó un daño innecesario a un paciente". Los incidentes pueden originarse en actos intencionados o involuntarios; se denomina infracción a un desvío deliberado de las normas o procedimientos operativos, mientras que el error es, por definición, siempre involuntario ${ }^{3}$.

Un incidente ocurre cuando un evento alcanza al paciente, y puede ser un incidente sin daño en que no hay un daño apreciable, o un incidente con daño, en que el evento alcanza al paciente y genera un daño, lo que también se conoce como evento adverso (EA).

Según el reporte del IOM, en EE. UU. ocurren más de 1 millón de EA prevenibles cada año, de los cuales aproximadamente 100.000 causarían daños graves en los pacientes, y entre 48.000 y 98.000 muertes podrían haber sido a causa de errores. Según estas cifras, los EA prevenibles causarían más muertes al año que accidentes vehiculares, cáncer de mama, o SIDA ${ }^{1}$. Estas cifras, consideradas estimaciones mínimas y máximas de la cantidad de muertes causadas por errores médicos, son extrapolaciones basadas en dos estudios. El estudio de práctica médica de Harvard, que analizó egresos hospitalarios en Nueva York, reportó que los EA ocurrían en 3,7\% de las hospitalizaciones, siendo $58 \%$ atribuibles a errores y de los cuales la mitad $(27,6 \%)$ eran atribuibles a negligencia ${ }^{4}$. Un estudio posterior realizado también en EE. UU., corroboró estos hallazgos, reportando una tasa de EA de $2,9 \%$, siendo atribuibles a errores $53 \%$, alrededor de $30 \%$ del total atribuibles a negligencia ${ }^{5,6}$. Cifras más recientes indican que en vez de disminuir su importancia, los errores médicos constituirían la tercera causa de muerte en EE. UU, dando cuenta de cerca de 400.000 muertes por año, cuadruplicando las cifras del $\mathrm{IOM}^{2}$. Un estudio chileno del año 2009 mostró una incidencia de EA en el sistema público de $6,7 \%$, de los cuales $84,7 \%$ eran prevenibles ${ }^{7}$; esta cifra casi duplica lo reportado en EE. UU. En la Tabla 1 realizamos un resumen de cifras de incidencia de eventos adversos a nivel mundial, junto al porcentaje de éstos que fueron considerados como prevenibles. Es llamativo que Latinoamérica concentre la mayor incidencia de EA (casi 20\%), siendo además la mayoría de éstos prevenibles. Las diferencias encontradas entre países pueden deberse a variados factores (sociodemográficos, culturales, económicos, pacientes con distintas co-morbilidades, complejidad de los centros de salud, entre otras), que escapan el objetivo de esta comunicación.

En el ámbito de los eventos adversos, aquellos causados por errores en medicina tienen componentes éticos particulares. No solamente pueden tener consecuencias físicas y psicológicas para los pacientes, para el sistema de salud y también a nivel económico, si no que pueden producir dudas, culpabilidad, vergüenza, estrés postraumático, miedo y ejercicio a la defensiva en el personal de salud $^{8}$. Además, según cifras de la Organización Mundial de la Salud (OMS), se ha estimado que producirían costos adicionales en la atención sanitaria de hasta 19 billones de dólares al año ${ }^{9}$ y pueden dañar la credibilidad de los servicios de salud cuando se hacen conocidos ${ }^{8,10}$. Por esto, existen dificultades por parte del personal de salud en comunicar los errores, argumentando probables daños innecesarios a los pacientes o aumento en el riesgo de demandas ${ }^{11}$. No obstante, estudios observacionales han mostrado que al revelar por completo los errores, hay menos demandas ${ }^{12-14}$.

\section{Opinión de los pacientes respecto del revelado de errores médicos}

Un estudio realizado en EE. UU. exploró las preferencias de los pacientes respecto al reporte de errores, demostrando que ellos quieren ser informados, y saber que las mejoras de calidad efectuadas debido a este aprendizaje servirán para evitar que lo que les sucedió le ocurra a otras personas. En consecuencia, prefieren que el reporte de 
Tabla 1. Resumen de tasas de eventos adversos y porcentaje de eventos adversos prevenibles en diversos estudios encontrados a nivel internacional

\begin{tabular}{|lcccc|}
\hline País & Año & Incidencia eventos adversos & Prevenibles & Referencia \\
\hline Latinoamérica & 2009 & $19,8 \%$ & $65 \%$ & 32 \\
\hline Australia & 1995 & $16,6 \%$ & $51 \%$ & 33 \\
\hline Nueva Zelanda & 2002 & $12,9 \%$ & $37,1 \%$ & 33 \\
\hline UK & 2006 & $10,9 \%$ & $\mathrm{~N} / \mathrm{E}^{*}$ & 33 \\
\hline Reino Unido & 2001 & $10,8 \%$ & $47,9 \%$ & 33,34 \\
\hline España & 2005 & $9,3 \%$ & $42,8 \%$ & 35 \\
\hline Canadá & 2004 & $7,5 \%$ & $37 \%$ & 33 \\
\hline EE. UU. & 1993 & $7,5 \%$ & $43,5 \%$ & 33,36 \\
\hline Canadá & 2004 & $5 \%$ & $\mathrm{~N} / \mathrm{E}^{*}$ & 37 \\
\hline Colombia & 2008 & $4,6 \%$ & $61 \%$ & 38 \\
\hline EE. UU. & 1991 & $3,8 \%$ & $\mathrm{~N} / \mathrm{E}^{*}$ & 33 \\
\hline EE. UU. & 2000 & $3,2 \%$ & $\mathrm{~N} / \mathrm{E}^{*}$ & 33 \\
\hline
\end{tabular}

Ordenados de mayor a menor tasa de eventos adversos. *N/E: No se cuantificó cuántos de los eventos adversos eran prevenibles en este estudio.

errores sea obligatorio, quieren saber que tanto el personal como las instituciones aceptan los errores frente a sus líderes, jefes y pares, y que los errores se reportan tan pronto como se detectan ${ }^{15}$. La experiencia de un abogado relata que, en la mayoría de los casos, lo que los pacientes realmente querían era sólo una explicación sincera de qué sucedió, y una disculpa ${ }^{16}$. Tal como lo muestran numerosos estudios, el no comunicar los errores a los pacientes afecta la confianza y satisfacción de los pacientes y aumenta las probabilidades de una demanda ${ }^{17-21}$.

Otro estudio americano evaluó las preferencias de padres de población pediátrica respecto al reporte y revelado de errores, validando también una amplia preferencia por el revelado de errores (99\%) y el reporte de los mismos a instancias disciplinarias (39\%); en 36\% de los casos el revelado del error disminuía las probabilidades de buscar apoyo legal por parte de los padres ${ }^{12}$. Es importante señalar que la raza/etnia, edad, género y nivel de educación de los pacientes no parecen influir de manera sustantiva en sus preferencias respecto de revelar los errores ${ }^{12}$. Sí hay diferencias en las preferencias de los padres respecto de revelar cuando hay daño (99\%) versus si no lo hay (77\%), pero las cifras son contundentes en mostrar la preferencia hacia revelar e incluso hacerlo también hacia el niño, dependiendo de la edad de éste ${ }^{22}$.

\section{Aspectos legales y normas internacionales respecto del reporte de errores}

La postura de sociedades médicas alrededor del mundo apunta hacia el reporte (al comité de calidad) y revelado (a los pacientes) de errores y efectos adversos, tal como se muestra en la Tabla 2. En Chile, la ley 20.584, que regula los derechos y deberes que tienen las personas en relación con acciones vinculadas a su atención en salud, tiene un párrafo específico relacionado con la seguridad en la atención de salud, cuyo Artículo $4^{\circ}$ señala que la persona tiene derecho a que se cumplan con los protocolos establecidos en materias tales como "errores en la atención en salud" y, en general, todos aquellos eventos adversos evitables según las prácticas comúnmente aceptadas. Dicha ley expresamente señala que la persona tiene "derecho a ser informada acerca de la ocurrencia de un evento adverso, independientemente de la magnitud de los daños que aquel haya ocasionado"23. Igualmente, han sido incluidas como componente de la acreditación institucional, tanto en atención 
Tabla 2. Recomendaciones de sociedades médicas del mundo referente al revelado de errores

\begin{tabular}{|c|c|c|}
\hline País & Asociación & Recomendación \\
\hline Australia & $\begin{array}{l}\text { Australian Council for Safety and Quality in } \\
\text { Health Care }\end{array}$ & $\begin{array}{l}\text { Recomienda revelado de errores luego de eventos } \\
\text { adversos }{ }^{39}\end{array}$ \\
\hline Canadá & Canadian Medical Association & Obliga revelado de errores ${ }^{40}$ \\
\hline Chile & Sociedad Chilena de Medicina Interna & Obliga revelado de errores ${ }^{41}$ \\
\hline EE. UU. & American Medical Association & $\begin{array}{l}\text { Obliga a informar al paciente de complicaciones que } \\
\text { puedan haber resultado de un error médico }{ }^{42,43}\end{array}$ \\
\hline Italia & Italian Code of Medical Ethics & $\begin{array}{l}\text { Deber ético de informar al paciente ante cada evento no } \\
\text { deseado y sus causas. Identificar, reportar eventos adver- } \\
\text { sos, casi errores y errores de procedimiento y diagnóstico }{ }^{44}\end{array}$ \\
\hline Nueva Zelanda & Medical Council of New Zealand & Obliga revelado de errores ${ }^{45}$ \\
\hline Reino Unido & National Health Service & $\begin{array}{l}\text { Obliga revelado de errores ante actos de negligencia u } \\
\text { omisión que causen daño } 46\end{array}$ \\
\hline Suecia & $\begin{array}{l}\text { Autoridad central de salud (Socialstyrelsen), } \\
\text { Autoridad disciplinaria (Ansvarsnämnden) }\end{array}$ & Obliga revelado de errores ${ }^{47}$ \\
\hline Suiza & Swiss Academy of Medical Sciences & $\begin{array}{l}\text { Dice que los médicos deben ser honestos y transparentes, } \\
\text { pero no establece obligación de reportar los errores, sí } \\
\text { recomienda esfuerzos educativos en relación al tema }{ }^{47}\end{array}$ \\
\hline
\end{tabular}

abierta como cerrada, medidas de prevención y vigilancia de errores, aunque no se establece el cómo deben funcionar ${ }^{24}$. En EE. UU., la Joint Commission on the Accreditation of Healthcare Organizations (JCAHO) requiere que exista un protocolo de revelación de resultados no anticipados (unanticipated outcomes), políticas que se han repetido en el Reino Unido, Canadá y Australia, enfatizando que estas recomendaciones tienen como foco el contribuir a identificar factores que contribuyen al error humano, más que a buscar responsabilidades ${ }^{25,26}$.

\section{Principios éticos y tensiones no resueltas}

De acuerdo a la evidencia presentada, las preferencias de los pacientes y familiares favorecen el reporte y revelado de errores, incluso cuando el error ocurrió sin consecuencias. Inicialmente, podemos considerar que la revelación del error es desde el punto de vista ético necesaria en una gran cantidad de casos para poder resolver los resultados adversos inesperados que se han provocado. En el contexto actual de la atención de salud, en donde se reconoce el consentimiento informado como un aspecto fundamental para poder dar inicio a la acción médica, las intervenciones necesarias para dar resolución a un EA requieren de una justificación en cuanto a la causa del problema, haciendo ineludible el revelado del error médico cuando éste fue la causa del evento. Por lo demás, existe una obligación de revelar la ocurrencia del error fundada también en el principio de autonomía del paciente, toda vez que se puede haber afectado el plan de cuidado trazado inicialmente ${ }^{27}$.

La necesidad de reporte y revelado no se limita solamente a los eventos adversos (entendidos, como se definieron al principio, como incidentes con daños), sino que también a aquellos eventos que se consideran en la nomenclatura de la OMS como cuasi incidentes, siendo aquellos en que el evento no alcanza al paciente. Éstos, en caso de que se hayan debido a un error médico, pueden nominarse casi errores, nomenclatura que se ha propuesto en la evidencia internacional del tema. Concordando con la opinión de pacientes, detallada en los estudios señalados, pacientes expuestos a errores, aun cuando éstos no hayan provocado daños, deben ser informados, no sólo en consideración al respeto a su autonomía, sino que también considerando que pueden jugar un rol importante en la prevención de futuros errores 
en su cuidado. De hecho, los estudios indican que es 300 veces más probable que se produzca un casi error que un error, cobrando relevancia su reporte al personal de salud y al propio paciente, con fines preventivos y de aprendizaje continuo ${ }^{11,26,28,29}$.

En cuanto al supuesto aumento en el estrés de los pacientes al revelar errores (lo que justificaría el mal llamado privilegio terapéutico de no revelar), se ha visto que cuando se revelan con honestidad y compasión por parte del profesional de la salud, y acompañados de una disculpa sincera, disminuye el estrés sobre el paciente ${ }^{11,29}$.

Tomando como base que un error médico puede dañar a un paciente, no revelarlo incurre en el riesgo de empeorar las consecuencias, y deberá ponderarse caso a caso, según las preferencias de las personas, cuál es la mejor conducta a seguir. Creemos que es válido que existan pacientes que indiquen expresamente a su médico que no desean obtener esta información, en los cuales sería legítimo no entregarla como una forma de respetar su autonomía. En caso contrario, el médico tiene una obligación prima facie de revelar el error. Cabe destacar que hacemos una distinción entre el revelar la información al paciente y el reporte del error al resto del equipo de salud, el cual vemos como imprescindible de hacer, para utilizarlo como experiencia de aprendizaje y contribuir a la mejora de la calidad de la atención.

El revelar respeta el principio de veracidad, el cual contribuye a promover la credibilidad en la profesión médica y el respeto entre profesionales y pacientes. La evidencia demuestra que conversar francamente con los pacientes, y contarles ya sean buenas o malas noticias, tiene un efecto beneficioso en la relación médico-paciente ${ }^{10,29}$. Del mismo modo, no revelar el error al paciente por temor a la reacción de éste o a una posterior demanda, significa que se colocan los intereses del médico por delante de los del paciente, siendo una violación al principio de beneficencia que constituye un quiebre de la relación fiduciaria en que se funda la práctica médica y de otras profesiones de la salud. El no revelar un error también implica que se pierde la posibilidad de ofrecer una compensación justa al paciente por el error que se cometió, cuya mínima expresión es una disculpa; esta justa compensación es la expresión del principio de justicia. Ocultar o no revelar errores artificialmente disminuye los costos reales del error médico, causando menos premura en el establecimiento de mecanismos para prevenirlos, lo cual termina afectando a pacientes futuros. Este tipo de faltas, en que los efectos de no revelar errores en forma sistemática y ordenada pueden causar desmedro de las condiciones de atención de salud que viven poblaciones futuras, puede considerarse una forma de maleficencia hacia generaciones futuras por parte de aquellos que ocultan el error.

No obstante, debemos reconocer que existen tensiones no resueltas respecto del revelado de los errores al paciente, y que muchas veces los médicos experimentan presiones por parte de los defensores de los derechos de los pacientes y expertos en bioética hacia el completo revelado de los errores y, por otra parte, los administradores y los encargados de los seguros de mala práctica esperan que los médicos revelen lo menos posi$\mathrm{ble}^{30}$. Este desacuerdo es mayor cuando se trata de errores que no han tenido consecuencias, puesto que expone al prestador a juicios de mala práctica tanto si revela como si no lo hace y luego el paciente se entera de la realidad. Si bien la mayoría de los médicos considera que los errores deben ser revelados al paciente, cuando estos ocurren se intentan ocultar, por considerar que así se protege la confianza en la relación médico paciente y que no se estaría causando daño al paciente, especialmente en la medida que el error no haya producido consecuencias, como en el caso de un casi error o un incidente sin daño. Pero seguir este curso de acción implica reconocer que el profesional está anteponiendo sus intereses por sobre los de su paciente ${ }^{29}$. Parte importante de evitar esto está dado por crear un ambiente en que se reconoce que los errores son cometidos en general por buenos profesionales, dedicados a su trabajo, pero que trabajan en ambientes imperfectos, con organismos biológicos complejos e impredecibles, y que están sometidos, con la mejor de sus intenciones, a presiones de intereses que a veces son contrapuestos en la sociedad que se desenvuelven.

\section{Conclusión}

Las razones expuestas apuntan a que el revelado de los errores médicos debe siempre ser ofrecido al paciente, mientras que el reporte de errores a los responsables del equipo de salud es mandatorio, para colaborar con el aprendizaje y la mejora continua de la atención en salud. Es 
posible que existan casos excepcionales en que no sea conveniente revelar los errores, como en patologías psiquiátricas severas, en quienes esta información podría producir un agravamiento de su cuadro, pero estos casos no constituyen la regla, y es posible que esos pacientes, además, estén bajo tutela de un representante, en cuyo caso el revelado de errores puede realizarse con la persona que lo representa. Sólo en casos muy específicos podría tener un efecto adverso en el paciente el revelado de errores y se puede plantear la necesidad de postergar la comunicación del error a un momento posterior.

Nuestra propuesta difiere de lo que es la norma actual en la Ley de Derechos y Deberes de los Pacientes, en donde solamente existe la obligación de revelar los EA que se hayan producido, que en la nomenclatura internacional implicaría solamente revelar aquellos errores que hayan producido daño. La actual norma chilena nos parece demasiado laxa por varios motivos. Primero, considera que el casi error es innecesario que se revele, ya que por definición no tiene consecuencias, y hemos presentado arriba las razones por lo que esto es inadecuado. Segundo, supone que los efectos adversos siempre pueden ser detectados, pero pueden haber efectos adversos que no fueron buscados al momento de cometerse un error, o efectos adversos poco comunes cuya ocurrencia no es usualmente buscada. Por último, la norma pareciera considerar que los efectos adversos son siempre inmediatos, en circunstancias en que muchos de ellos pueden ocurrir meses o incluso años después, confundiéndose su origen con posibles causas no relacionadas al error que las produjo.

Es necesario revisar experiencias internacionales de programas de revelado de errores para determinar qué modelos son aplicables a nuestro contexto, para posteriormente documentar experiencias nacionales innovadoras que permitan mejorar esto en nuestro país. A modo de ejemplo, existe documentación de programas de resolución de conflictos que incluyen aspectos como pedir disculpas, ofrecer explicaciones $y$, cuando sea apropiado, ofrecer compensaciones razonables a los pacientes, manteniendo una vigorosa defensa del proveedor de salud cuando los análisis de causales de un resultado adverso muestren que se mantuvo un adecuado nivel de cuidado ${ }^{31}$.

Comprendemos las preocupaciones con respecto a la judicialización de la práctica médica, lo cual en muchas situaciones generará dificultades en el equipo de salud con respecto a reportar y revelar errores. Creemos, sin embargo, que por las razones presentadas anteriormente, esta conducta de revelado y reporte debiese ser en general obligatoria.

Por último, consideramos necesario realizar más estudios en nuestro medio, para ver si se repite la tendencia encontrada en otros países, donde al revelar los errores disminuye la intención de demanda por parte de los pacientes, algo que podría hacer más fácil transparentar estos tristes episodios.

\section{Referencias}

1. Kohn L, Corrigan J, Donaldson M, eds. Committee on Quality of Health Care in America, Institute of Medicine. To Err is Human: Building a Safer Health System. Washington, DC: National Academy Press, 2000. Disponible en http://www.nap.edu/catalog/9728.html. (consultado el 15 de agosto de 2015).

2. Makary MA, Daniel M. Medical error-the third leading cause of death in the US. BMJ 2016; 353: i2139.

3. World Health Organization. Marco Conceptual de la Clasificación Internacional para la Seguridad del Paciente. Versión 1.1. Informe técnico definitivo. 2009.

4. Leape LL, Brennan TA, Laird N, Lawthers AG, Localio $\mathrm{AR}$, Barnes BA, et al. The nature of adverse events in hospitalized patients. Results of the Harvard Medical Practice Study II. N Engl J Med 1991; 324 (6): 377-84.

5. Thomas EJ, Studdert DM, Burstin HR, Orav EJ, Zeena $\mathrm{T}$, Williams EJ, et al. Incidence and types of adverse events and negligent care in Utah and Colorado. Med Care 2000; 38 (3): 261-71.

6. Thomas E, Studdert D. Costs of medical injuries in Utah and Colorado. Inquiry 1999; 36 (3): 255.

7. Álvarez N. Resumen del Estudio Nacional de incidencia Eventos Adversos en hospitales públicos de Chile, 2009. Disponible en: http://pacienteseguro.cl/Contenidos/ RESUMEN.pdf. (consultado el 11 de enero de 2016).

8. Gómez Córdoba A, Espinosa A. Dilemas éticos frente a la seguridad del paciente. Cuidar es pensar. Aquichan 2006; 6 (1): 54-67.

9. World Health Organization. 10 facts on patient safety. 2014 Disponible en: http://www.who.int/features/factfiles/patient_safety/patient_safety_facts/en/. (consultado el 21 de agosto de 2015).

10. Wolf Z, Hughes R. Error Reporting and Disclosure. In: Hughes RG, editor. Patient Safety and Quality: An 
Evidence-Based Handbook for Nurses. Rockville (MD): Agency for Healthcare Research and Quality (US); 2008 Apr. Chapter 35. Disponible en: http://www.ncbi.nlm. nih.gov/books/NBK2652/. (consultado el 15 de agosto de 2015).

11. Carvallo A. Consideraciones éticas sobre el error en medicina. Rev Med Chile 2001; 129 (12): 1463-5.

12. Hobgood C, Tamayo-Sarver JH, Elms A, Weiner B. Parental preferences for error disclosure, reporting, and legal action after medical error in the care of their children. Pediatrics 2005; 116 (6): 1276-86.

13. Schwappach DL, Koeck CM. What makes an error unacceptable? A factorial survey on the disclosure of medical errors. Int J Qual Health Care 2004; 16 (4): 317-26.

14. Mazor KM, Simon SR, Yood RA, Martinson BC, Gunter MJ, Reed GW, et al. Health plan members' views about disclosure of medical errors. Ann Intern Med 2004; 140 (6): 409-18.

15. Gallagher TH, Waterman AD, Ebers AG, Fraser VJ, Levinson W. Patients' and physicians' attitudes regarding the disclosure of medical errors. JAMA 2003; 289 (8): 1001-7.

16. Wu AW. Handling hospital errors: is disclosure the best defense? Ann Intern Med 1999; 131 (12): 970-2.

17. Witman AB, Park DM, Hardin SB. How do patients want physicians to handle mistakes? A survey of internal medicine patients in an academic setting. Arch Intern Med 1996; 156 (22): 2565-9.

18. Vincent C, Young M, Phillips A. Why do people sue doctors? A study of patients and relatives taking legal action. Lancet 1994; 343 (8913): 1609-13.

19. Hingorani M, Wong T, Vafidis G. Patients' and doctors' attitudes to amount of information given after unintended injury during treatment: cross sectional, questionnaire survey. BMJ 1999; 318 (7184): 640-1.

20. May T, Aulisio MP. Medical malpractice, mistake prevention, and compensation. Kennedy Inst Ethics J 2001; 11 (2) : 135-46.

21. Vincent CA, Coulter A. Patient safety: what about the patient? Qual Saf Health Care 2002; 11 (1): 76-80.

22. Matlow AG, Moody L, Laxer R, Stevens P, Goia C, Friedman JN. Disclosure of medical error to parents and paediatric patients: assessment of parents' attitudes and influencing factors. Arch Dis Child 2010; 95 (4): 286-90.

23. Ley 20.584. Regula los derechos y deberes que tienen las personas en relación con acciones vinculadas a su atención en salud. Ed. Ministerio de Salud. Promulgada el 13 de abril de 2012. Biblioteca del Congreso Nacional de Chile. Disponible en http://bcn.cl/1m0do.

24. Superintendencia de Salud de Chile. Manuales de Acreditación para Prestadores Institucionales de Salud.
2009. Disponible en: http://www.supersalud.gob.cl/568/ w3-article-4530.html. (consultado el 15 de agosto de 2015).

25. Hammami MM, Attalah S, Al Qadire M. Which medical error to disclose to patients and by whom? Public preference and perceptions of norm and current practice. BMC Med Ethics 2010; 11: 17.

26. Barach P, Small SD. Reporting and preventing medical mishaps: lessons from non-medical near miss reporting systems. BMJ 2000; 320 (7237): 759-63.

27. Jones JW, McCullough LB. Transgression confession: ethics of medical error disclosure. J Vasc Surg 2013; 58 (6): 1697-9.

28. Rosselot E. Errores en medicina. Rev Med Chile 2001; 129 (12): 1455-8.

29. Edwin A. Non-disclosure of medical errors an egregious violation of ethical principles. Ghana Med J 2009; 43 (1): 34-9.

30. Blood J. Ethical responsibility and treatment errors. Br Dent J 2014; 216 (2): 59-60.

31. Mello MM, Boothman RC, McDonald T, Driver J, Lembitz A, Bouwmeester D, et al. Communication-and-resolution programs: the challenges and lessons learned from six early adopters. Health Aff (Millwood) 2014; 33 (1): 20-9.

32. Aranaz-Andrés J, Aibar-Remón C. Estudio IBEAS: Prevalencia de efectos adversos en hospitales de Latinoamérica. Madrid: Gobierno de España, Ministerio de Sanidad, Política Social e Igualdad. 2010.

33. de Vries EN, Ramrattan MA, Smorenburg SM, Gouma DJ, Boermeester MA. The incidence and nature of in-hospital adverse events: a systematic review. Qual Saf Health Care 2008; 17 (3): 216-23.

34. Neale G, Woloshynowych M, Vincent C. Exploring the causes of adverse events in NHS hospital practice. J R Soc Med 2001; 94 (7): 322-30.

35. Ministerio de Sanidad y Consumo de España. Estudio Nacional sobre los Efectos Adversos ligados a la Hospitalización. ENEAS 2005. Informe. Elche: Dirección General de la Agencia de Calidad del Sistema Nacional de Salud, Secretaría General de Sanidad; 2006. Número de informe: 351-06-009-2. Disponible en: http://www. msssi.gob.es/organizacion/sns/planCalidadSNS/pdf/ excelencia/opsc_sp2.pdf. (consultado el 11 de enero de 2016).

36. Bates DW, O'Neil AC, Petersen LA, Lee TH, Brennan TA. Evaluation of screening criteria for adverse events in medical patients. Med Care 1995; 33 (5): 452-62.

37. Forster AJ, Asmis TR, Clark HD, Al Saied G, Code CC, Caughey SC, et al. Ottawa Hospital Patient Safety Study: incidence and timing of adverse events in patients admi- 
tted to a Canadian teaching hospital. CMAJ 2004; 170 (8): 1235-40.

38. Gaitán-Duarte H, Eslava-Schmalbach J, Rodríguez-Malagon N, Forero-Supelano V, Santofimio-Sierra D, Altahona $\mathrm{H}$. Incidencia y Evitabilidad de Eventos Adversos en Pacientes Hospitalizados en tres Instituciones Hospitalarias en Colombia, 2006. Rev Salud Pública (Bogotá) 2008; 10 (2): 215-26.

39. Australian Commission on Safety and Quality in Health Care. Australian Open Disclosure Framework. Sydney: ACSQHC; 2013. Disponible en http://www. safetyandquality.gov.au/wp-content/uploads/2013/03/ Australian-Open-Disclosure-Framework-Feb-2014.pdf. (consultado el 15 de agosto de 2015).

40. Canadian Medical Association. CMA Code of Ethics. 2004 Disponible en: http://policybase.cma.ca/dbtw-wpd/PolicyPDF/PD04-06.pdf. (consultado el 15 de agosto de 2015).

41. Mena NP. Error médico y eventos adversos. Rev Chil Pediatr 2008; 79 (3): 319-26.

42. American Medical Association. Principles of Medical
Ethics. 2001. Disponible en: http://www.ama-assn.org/ ama/pub/physician-resources/medical-ethics/code-medical-ethics/principles-medical-ethics.page. (consultado el 15 de agosto de 2015).

43. AMA. Council on Ethical and Judicial Affairs and Southern Illinois University School of Law. Code of Medical Ethics, Annotated Current Opinions. Chicago, IL: AMA; 1994.

44. Turillazzi E, Neri M. Medical error disclosure: from the therapeutic alliance to risk management: the vision of the new Italian code of medical ethics. BMC Med Ethics 2014; 15: 57.

45. Fyfe J, Connolly A, Bond B. Informed consent. Chapter 10 in St George IM, editor. Cole's medical practice in New Zealand, 12th edition. Wellington: Medical Council of New Zealand, 2013. p. 104.

46. Dyer C. NHS staff should inform patients of negligent acts. BMJ 2003; 327 (7405): 7.

47. de la Ruee R. [Medical malpractice and patients' rights in Sweden] Schweizerische Ärztezeitung 2007; 88 (46): 1958-60. 